\title{
Transcript-based computer animation of movement: Evaluating a new tool for nonverbal behavior research
}

\author{
GARY BENTE, ANITA PETERSEN, NICOLE C. KRÄMER, and JAN PETER DE RUITER \\ University of Cologne, Cologne, Germany
}

\begin{abstract}
A new approach for the use of computer animation in experimental nonverbal research is introduced. The method was evaluated in a pilot study comparing video recordings of movement in dyadic interactions with computer animations based on transcripts of the behavior, to determine whether similar impression effects could be obtained. At the core of our development is a software tool allowing for the conversion of so-called position time-series protocols of movement into animation scripts for a professional computer animation platform. Our software combines computer-assisted movement transcription and editing with state-of-the-art3-D animation technology. We present empirical evidence indicating remarkable overall correspondence between video recordings and computer animations. Due to the lack of facial activity in the computer animations, a decline in visual attention for the face area could be observed, which did not, however, affect the impression ratings.
\end{abstract}

Although most researchers agree that nonverbal behaviors, such as gestures, body and head movements, postures, and so on, play an important role in human faceto-face interaction (Argyle, 1972; Davis, 1972; Grammer, Kruck, \& Magnusson, 1998; Mehrabian, 1969), the experimental analysis of the interpersonal effects that are exerted by particular nonverbal patterns seems still in its infancy. As we pointed out earlier, major difficulties in preliminary research were related to the problem of experimental control over the independent variables-that is, insertion or manipulation of specific behavioral cues in the stimulus material under investigation (Bente, Feist, \& Elder, 1996). In particular, the common practice of instructing confederates of the experimenter or professional actors to vary particular aspects of their behavior did not provide reliable stimulus control. This is because of the many interactions among different nonverbal channels (see Lewis, Derlega, Shankar, Cochard, \& Finkel, 1997) and different features of appearance. Approaches that use photo retouch techniques, elastic dummies, or hand drawings allow for better control, but are restricted to static aspects of nonverbal behavior (Frey, Hirsbrunner, Florin, Daw, \& Crawford, 1983; Schouwstra \& Hoogstraten, 1995; Trautner, 1991). A promising alternative for the experimental analysis of dynamic features was suggested by Berry, Kean, Misovich, and Baron (1991). Based on a technique referred to as "quantization" (see

This research was supported by Grant BE 1745/2-1 from the Deutsche Forschungsgemeinschaft (DFG, German research association). Correspondence should be addressed to G. Bente, Department of Psychology, University of Cologne, Bernhard-Feilchenfeld-Strasse 11, 50969 Cologne, Germany (e-mail: bente@ uni-koeln.de).
Harmon, 1973; Morrone, Burr, \& Ross, 1983), standard videotapes are electronically distorted in a postproduction process to eliminate the recognition of most features of physical appearance. While this method is to some degree useful to control for the influence of physical appearance on person perception, it still lacks the important dimension of systematic experimental stimulus control. As Berry (1990) pointed out, in order to solve the problem, "the precise nature of the stimulus information communicating a given characteristic needs to be described. And that stimulus quality of interest needs to be manipulated in some fashion in order to demonstrate that this is indeed the effective stimulus underlying a given percept" (p. 150). Ideally, this would imply finding a digital representation of behavior that is open to analysis and experimental manipulation, and finding a way to retranslate these data into lifelike moving objects that can serve as social stimuli in observation studies. As a possible solution to the problem, we suggested the systematic use of graphical computer animation of nonverbal behavior, based on so called position time-series protocols transcribed from video-documented interactions behavior (Bente, 1989). Due to restrictions of the available hardware and software at that time, our first programs were restricted in scope, only allowing for the animation of a simple wire-frame model of the human head that could be overlaid on static images of various body models (see Bente et al., 1996, for an application). Recent advances in technology have facilitated the application of this methodology to dimensional extensions and more sophisticated computer graphic animations. For example, Kempter (2000) presented software for the editing and experimental computer animation of whole-body movement based on Open GL. The program is, however, restricted to 
the animation of a low-resolution 3-D model of a humanlike dummy. In fact, it is hardly possible to provide all the advanced modeling and animation features of a professional software platform (such as exchanging different body models, e.g., female and male ones, weighing polygon mashes to generate realistic changes of the skin when moving a part of the skeleton, etc.) in an isolated solution set up for scientific use only.

Against this background, we decided to integrate the advantages of a professional computer animation tool into our own approach. The present paper describes the basic principles of the new instrumentation and presents data from a preliminary evaluation experiment. This experiment addresses the question of which differential effects computer animations of human movement behavior exert on the observers' visual attention and interpersonal evaluation as opposed to video recordings of the same behavior. This comparison is necessary to validate future applications of this methodology in nonverbal research. No specific hypotheses were advanced with respect to the effects of particular movement patterns. Instead, we focused on technical variables necessary for the minimal requirements in animation quality and display techniques to capture realistic human movement. We also extended the range of dependent variables from subjective impression ratings to objective measurements of gaze direction to account for possible differences in visual attention and their influence on person perception. A variation within the computer animation condition (i.e., wire-frame model and surface model) was included in the experiments to determine possible influences of the animation quality on the person perception process.

\section{METHOD}

\section{General Method for 3-D Computer Animation of Movement Behavior}

As has been shown earlier, the availability of an efficient description methodology is essential for experimental computer animation of movement in particular (Bente, 1989). Whenever computer animations are based on recordings of real-life interactions, a coding language is needed that permits the transcription of complex movement patterns from video into data protocols that adequately reflect the complexity and dynamics of the recorded behavior. Since the coding language constitutes the interface between human observer and computer animation platform, it has to meet the specific requirements of both elements in this sociotechnical system. Most of the professional 3-D software tools or graphic languages share a common geometry, describing an object's spatial attributes in terms of translations and rotations along the axes of a 3 -D coordinate system. Although the spatial reference of the coordinate systems and the order in which rotations along the axes are performed might be different for these programs, the various data formats can easily be translated from one standard into another. On first glance, it may seem appropriate to refer to the generic 3-D geometry of any computer animation software when constructing a coding language for movement behavior. However, a closer examination reveals that it is very difficult for a human observer to determine the three rotation angles that lead to a particular posture of a body part. This problem is also described by professionals in the computer animation business using the so-called three-finger coordinate system to create a figurative imagination of possible rotation effects. In fact, 3-D animators mostly rely on a trial-and-error strategy, successively changing one or another rotation angle by numeric data entry or mouse drag and then checking the outcome. The confusion that can be observed during this operation is somehow puzzling, since it appears to be a minor problem for even laypeople to describe a given head position of a person on a photograph or a still video picture in terms of the degree of head lift, head rotation, and sideward tilt. It is this perceptual capacity upon which phenotypical coding strategies like the Bernese Coding System (BCS) for movement behavior are based (see Bente, 1989). The BCS (see Frey et al., 1983; Hirsbrunner, Frey, \& Crawford, 1987) is based on the principle of so-called position time-series notation. Movements are conceptualized as a time series of positions: The Bernese system is constructed as a kind of multidimensional alphabet, by means of which each source of variation (e.g., head, trunk, hands) is "spelled" separately in a position time-series protocol. Assigning numerical codes to the spatial deviations of the various body parts from predefined base positions, the BCS allows for a detailed and reliable transcription of video-recorded movement behavior into high-resolution data protocols (Frey \& Pool, 1976). The coding is done on an ordinal scale level. Although within the BCS the orientations of the various body parts are described with reference to a 3 -D coordinate system, systematic tests have shown that there is no direct correspondence between the BCS and the rotation logic of the available professional animation tools (see Leuschner, 1999). It became apparent that the implicit geometry of the BCS has to be understood in terms of projection angles of an object's local axes rather than in terms of generic rotation angles. Additionally, some of the coding dimensions in the BCS (i.e., the positions of hands and feet) are not all referring to rotation angles of joints but are coded as translations relative to specific fix points. Starting from this implicit geometry of the BCS, a software module has been developed-ICARUS (Interactive Coding, Analysis, and Rendering Utilities; Bente, Petersen, \& Krämer, 1999; Buschmann \& Krämer, 1999) - which supports interactive coding in the BCS standard and data conversion from BCS into generic rotation angles used by a professional 3-D animation platform. The program SoftImage3D was chosen as the animation platform for three major reasons: First, the software package already contains a sophisticated human body model consisting of an anthropomorphic skeleton and a realistic surface model of a male, which could easily be modified according to the requirements of our own study. Second, the program supplies a channel driver utility to import external data protocols. Third, SoftImage 3D contains a special real-time rendering window that could be used for immediate feedback during editing of the movement data. Combining this technology with our own converter module, we were able to produce accurate computer animation replications of videotaped nonverbal behavior and at the same time retain full access to descriptive movement data for the purpose of analysis and experimental variation of specif ic behavior dimensions (e.g., lateral head tilt).

As can be seen in Figure 1, the user interface of ICARUS shows the Bernese data protocols and the corresponding transformation in rotation angles of the data in different windows. Both allow for coding the given positions of various body parts as defined in the BCS. A real-time window gives immediate feedback of the coding process, which can be compared to the videotaped behavior. Additionally, BCS data coded elsewhere can be imported. Another feature of ICARUS is the availability of phenotypical codes (based on BCS) that can be edited by hand to create variations of the nonverbal behavior of interest (see Bente, 1989). Specif ic aspects of behavior, like postures, movements of head, body, or hands, or general movement qualities such as speed, acceleration, or complexity can be systematically controlled modifying the relevant numerical entries in the raw data. After finishing the coding or editing process, it is necessary to convert these data into the logic of the animation software. ICARUS converts the Bernese data (re- 


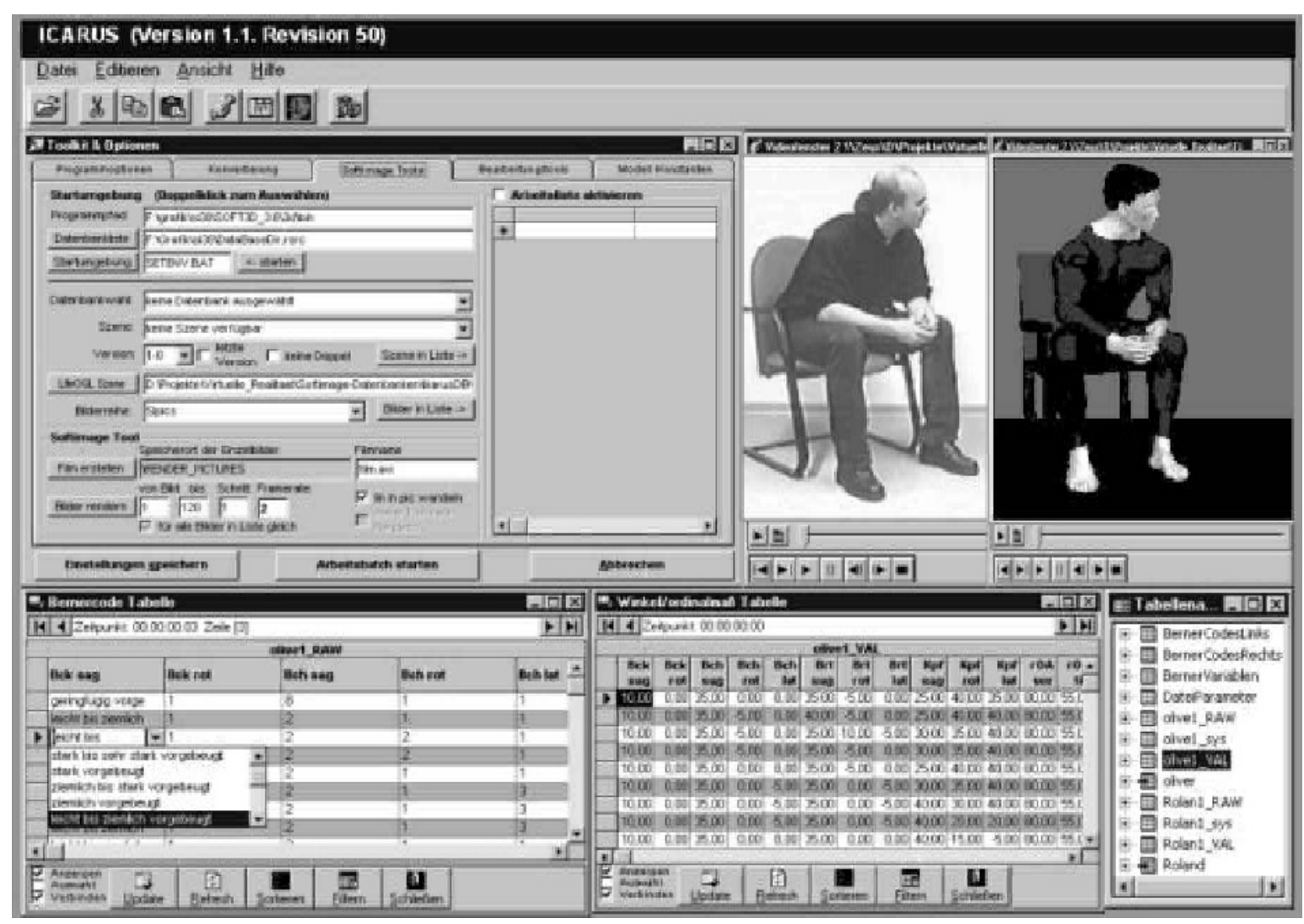

Figure 1. Graphical user interface of ICARUS (Interactive coding, analysis, and rendering utilities).

ferring to projection angles) into a channel-driver file, which is readable for the animation tool SoftImage3D (based upon a 3-D coordinate system).

\section{Stimulus Material}

A role-play interaction in which a disciplinary problem between a supervisor and an employee had to be discussed was chosen as stimulus material for our study. This situation causes some degree of emotional involvement in the actors and also contains explicit definitions of social roles and power hierarchy. Both aspects should guarantee a certain nonverbal activity level and evoke pronounced and distinctive nonverbal patterns for both actors. The role play was videotaped and $1 \mathrm{~min}$ of interaction was transcribed according to the principles of the BCS using the ICARUS software. Coding was done with a temporal resolution of $5 \mathrm{~Hz}$. Data then were interpolated to a rate of $25 \mathrm{~Hz}$ using the key-frame procedure of SoftImage3D and then rendered using the wire-frame model as well as the surface model. Both computer animation sequences were stored on hard disk in AVI format. The original video was digitized by means of a standard frame grabber and also stored on hard disk in AVI format. The different presentation modes for our study are shown in Figure 2.

\section{Experimental Design and Setting}

The sophistication of computer-generated stimuli was varied as can be seen in Figure 2. A reduced hidden-line wire-frame model was compared with a more realistic surface model with skin and clothes. Two display technologies were used in this study: (1) a binocular head-mounted display (HMD; PAL: $640 \times 480$ pixels) and (2) a large-screen LCD projector (XVGA: $1024 \times 768$ pixels). The HMD allowed the integration of an eye tracking system to mea- sure differences in the focus of visual attention when comparing video stimuli with computer animations. Because our computer animations did not include facial activity, it was hypothesized that shifts in visual attention would occur from the head to more active areas of body movement (e.g., hand gestures). In this case, we had to examine whether these shifts in gaze were also accompanied by differences in the impression ratings when compared with the video condition. Experiments using the HMD had to be run sequentially in single-subject mode. With respect to the economy of future studies, a group condition was included in the experimental design to determine possible audience effects on impression formation. A large-screen LCD projector was chosen for the group sessions, allowing free sight for up to 12 participants seated in a small room. To separate the influence of experimental setting (single vs. group) and display technology (HMD vs. LCD projector), a third experimental condition was included in our design using the LCD projector within a single-subject procedure. Table 1 gives an overview of the experimental conditions.

\section{Participants}

Sixty-four female and 32 male participants, $16-59$ years $(M=$ 31.9; $S D=9.6$ ), were recruited from different courses at the University of Cologne and from a job creation program. The participants were randomly assigned to one of the nine experimental groups.

\section{Dependent Measures}

The Positive and Negative Affect Schedule (PANAS; Krohne, Egloff, Kohlmann, \& Tausch, 1996; Watson, Clark, \& Tellegen, 1988) was used to measure the observers' attributions of emotional states and interpersonal attitudes toward both actors on the screen. 


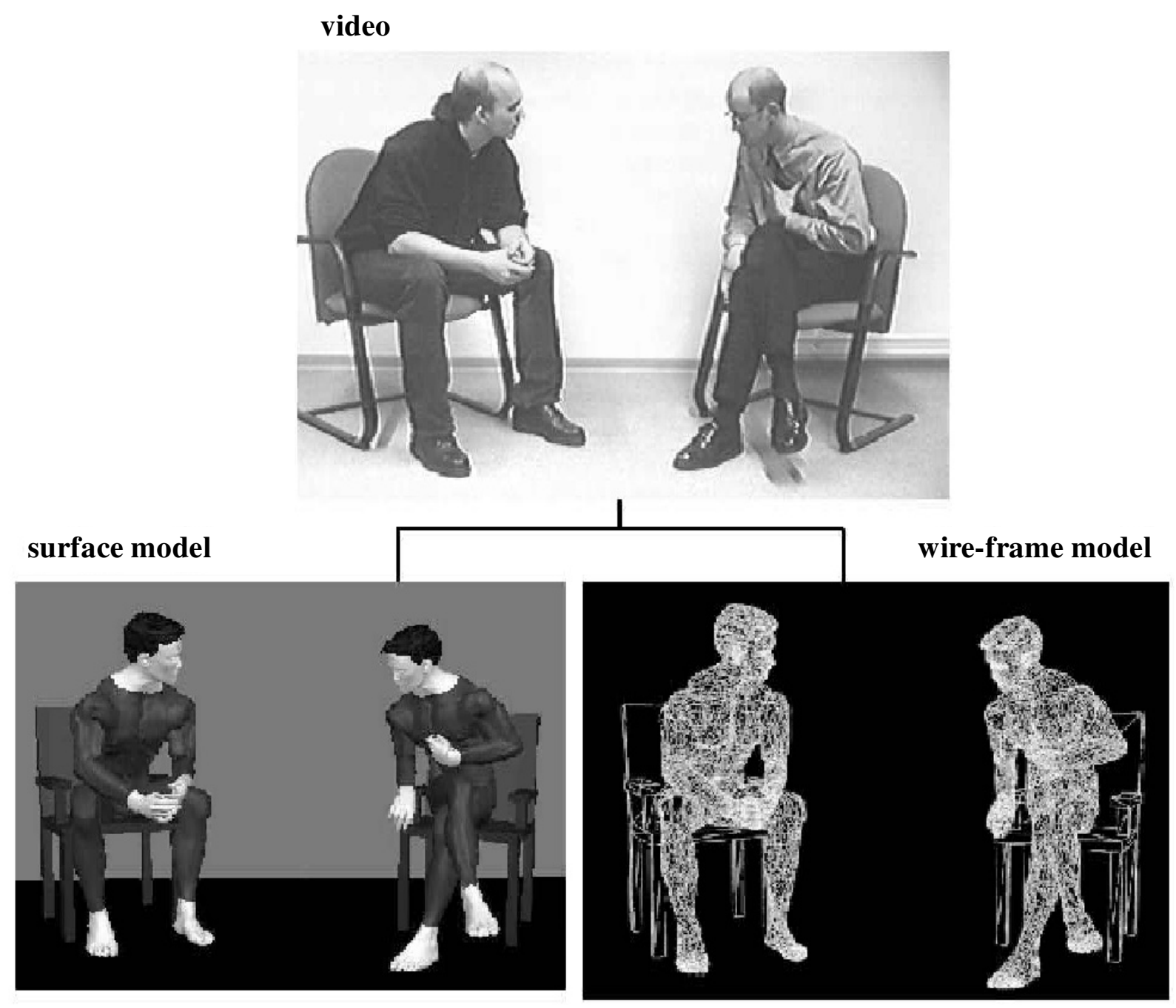

Figure 2. Examples of stimulus presentations in video and two computer animation conditions.

The following PANAS items were used with a 5-point scale $(1=$ not at all; 5 = extremely): active, interested, excited, strong, inspired, proud, enthusiastic, alert, determined, attentive, distressed, upset, guilty, scared, hostile, irritable, ashamed, nervous, jittery, and afraid. Although developed for self-assessment of emotional states, the system provides an item list that can be used for the judgment of other social stimuli as well. We also included three additional questionnaire items (Which person do you consider more sympathetic? Which person do you consider more active? Which person do you consider dominant?) asking for direct comparison of both actors according to the basic socioemotional dimension's "evaluation," "activity," and "potency" (see Mehrabian, 1969, 1970) and four items to assess the personal preferences of the observer for either one of the actors (Which person did you pay more attention to? Which person's perspective could you take more easily? Which person did you identify with? Which person would you prefer to talk to?).

Measurements of gaze direction were taken by means of the IOTA VideoEyeTrace-System, integrated in a binocular HMD for stimulus presentation (see above). The video-based system allows for the real-time detection of eye positions with a temporal resolution of $50 \mathrm{~Hz}$ and a spatial resolution below one angle degree (see http://www.iota.se; Bente et al., 1999). Due to the headset presen- tation, the stimulus remains in a stable position relative to the eyes of the observer; thus the system is very tolerant of head movements and no recalibrations are necessary even during longer measurement periods. For further analysis, the stimulus area was divided into six sections representing the right and left sides of the screen (the two actors) and three subdivisions for each actor: (1) upper area: shoulders upward (head movement and facial activity), (2) middle area: seat of the chair to shoulders (body, arm, and hand movements, i.e., gestural activity), and (3) lower area: seat of the chair to floor (leg and foot movement). As a global indicator of visual attention, the overall time of gaze contact with each of the six areas was calculated as a percentage of the total observation time.

Table 1

Experimental Design

\begin{tabular}{llccc}
\hline & & \multicolumn{3}{c}{ Presentation Modes } \\
\cline { 3 - 5 } Setting & Display Type & Video $(n)$ & Surface $(n)$ & Wire Frame $(n)$ \\
\hline \multirow{3}{*}{ Single } & Head-mounted display & 10 & 10 & 10 \\
Group & LCD projection & 10 & 10 & 10 \\
\hline
\end{tabular}




\section{Procedure}

Observers were seated in a test room equipped as a small-size cinema either alone or in groups of 12 . They were instructed to watch the stimulus sequence carefully and rate their impressions of the actors later on. The 1-min interaction sequences were then presented without sound in all conditions, either via binocular headset or via large screen projector. After stimulus presentation, the adjective list and the questionnaire were handed out to the participants to be filled out immediately.

\section{RESULTS}

Analyses of variance (ANOVAs) were used to trace significant discrepancies in the impression ratings between the experimental conditions. Differences in dichotomous questionnaire data were analyzed by means of chi-square tests. The alpha level for all tests was set to .01 (two-tailed).

\section{Influence of Display Technique and Experimental Setting on Person Perception}

ANOVAs for impression ratings and comparative evaluations of the interactors did not reveal any systematic influences of either display technique or social setting variables on person perception. Neither display mode (HMD vs. LCD projector) nor number of participants (single subject vs. group) affected the observers' judgments on any of the evaluative scales. Since gaze direction could be measured only in the single-subject HMD condition, there are no data available on setting effects for this variable.

\section{Focus of Visual Attention in Video and Computer Animation Conditions}

Analysis of gaze direction indicated that there was an equilibrium of visual attention for both screen actors, which was not affected by the presentation modes: Temporal distribution of gaze contact with left versus right person on the screen was $49.66 \%$ versus $50.12 \%$ in video mode, $48.14 \%$ versus $51.13 \%$ in surface mode, and $51.93 \%$ versus $47.80 \%$ in wire-frame mode (differences from $100 \%$ are due to measurement dropouts, e.g., eye blinks).

Significant differences, however, occurred for the temporal distribution of gaze over the three body areas. The percentage of time in which observers looked at the upper body areas (head movement and facial activity) dropped from $75.75 \%$ in the video condition to $56.23 \%$ in the surface-model condition and $51.05 \%$ in the wireframe condition, respectively $[F(2)=7.675, p=.003]$. Complementarily, visual attention increased for the middle part of the body (mainly gestural activity) from $21.74 \%$ to $41.06 \%$ and $45.09 \%$, respectively $[F(2)=$ $7.574, p=.003]$. Visual attention for the lower body areas showed no significant difference (1.38\% vs. $1.44 \%$ vs. $2.69 \%$ ). Figure 3 demonstrates the significant differences in visual attention for both stimulus persons separately. As can be seen, the remarkable shift in visual attention from the head area to the gestural area (trunk and upper extremities) occurred for both stimulus persons. Neither the difference between the stimulus persons that this figure shows nor the difference among both computer animations reached significance.

\section{Impression Formation Based on Video Versus Computer-Animated Stimuli}

Despite the marked differences in gaze direction, only a few differences occurred between emotional impression ratings based on video presentations and those based on computer animation sequences. Separate ANOVAs for both stimulus persons and the 20 items of
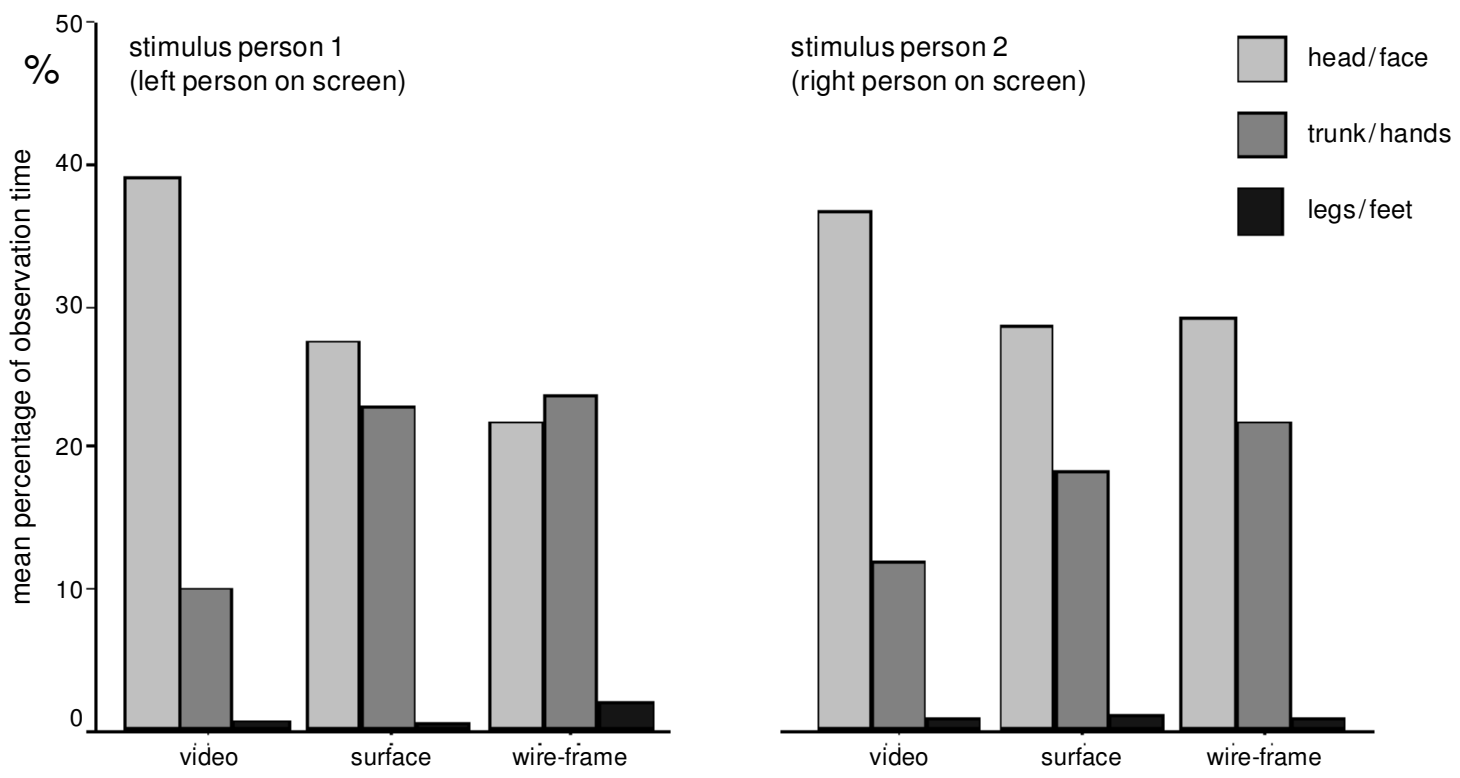

Figure 3. Temporal distribution of observers' gaze direction. 
the PANAS revealed only two significant differences, namely for the item upset for Stimulus Person $1[F(2)=$ $9.240, p=.000]$ and the item enthusiastic for Stimulus Person $2[F(2)=5.568, p=.005]$. Post hoc tests (Scheffé) indicated that Stimulus Person 1 was perceived to be more upset in the video condition $(M=2.74, S D=1.15)$ only when compared with the wire-frame model condition $(M=1.85, S D=0.74, p=.000)$, and Person 2 was perceived to be more enthusiastic in the surface-model condition $(M=1.27, S D=0.72)$ only when compared with the wire-frame condition $(M=1.88, S D=1.09, p=$ $.017)$. Figure 4 demonstrates the remarkable correspondences among the impression ratings based on video and computer animation stimuli.

\section{Comparative Evaluation of Stimulus Persons}

Chi-square tests were run for the comparative evaluation items to test differences in preference for one of both stimulus persons between video and computer animation modes. Preference choices in the video condition were treated as expected frequencies, and the preference choices in either one of the computer animation modes were treated as observed frequencies. Expected frequencies were corrected for the number of observers in the experimental group. The frequency distributions for all preference choices, and the results of chi-square tests are given in Table 2 . As can be seen, only 2 of the 14 chisquare tests showed significant differences. The comparative rating of activity showed an inverse relation in the wire-frame mode in relation to video, and the definite dominance rating for the left person that occurred in the video condition was leveled in the surface-model condition. The first effect was probably due to the lack of facial activity, most evident for the wire-frame mode, with the consequence that body movement received more attention for the judgment of activity. Indeed, the

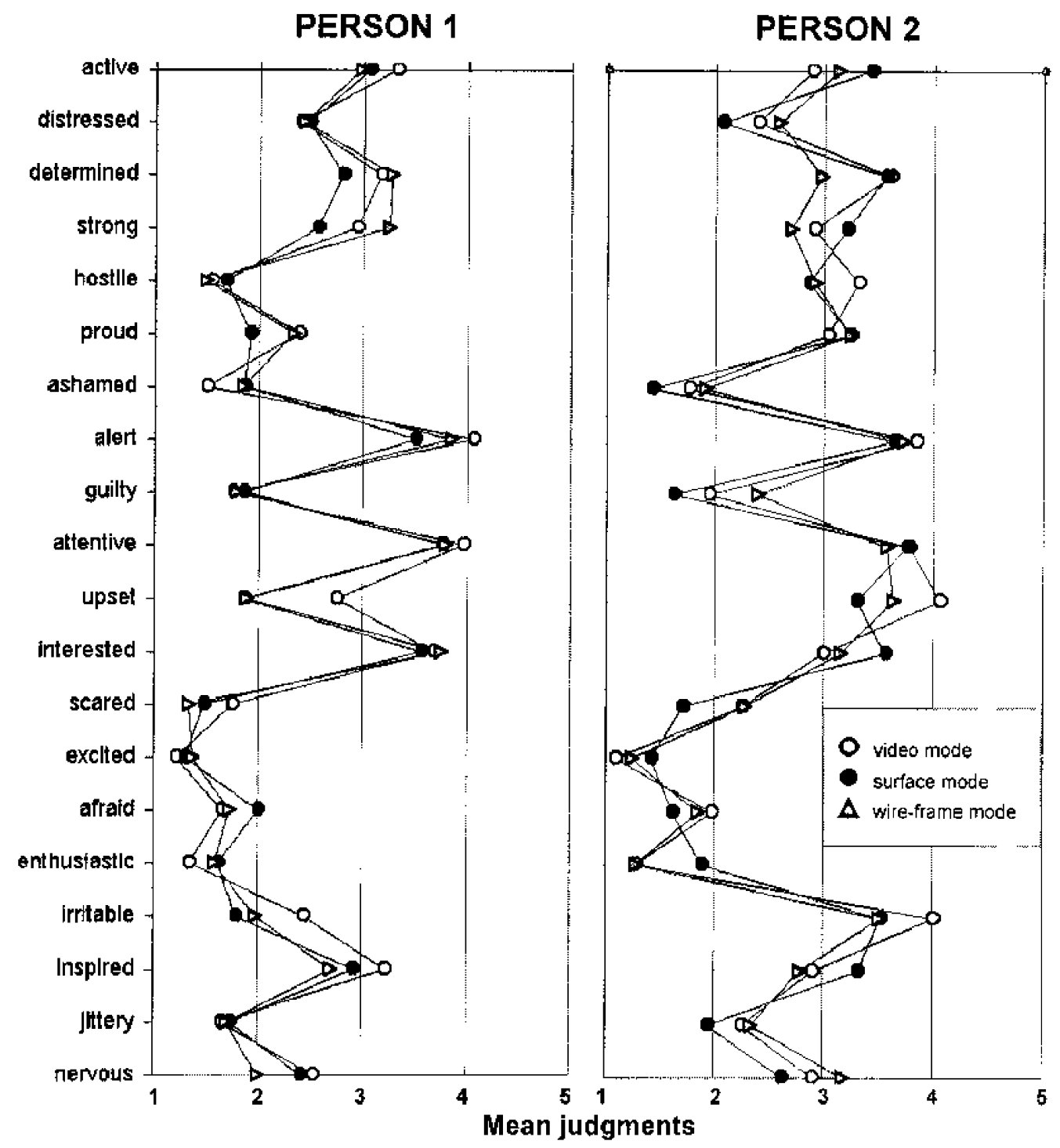

Figure 4. Impression rating profiles for stimulus persons in video and two computer animation modes. 
Table 2

Frequency Distribution of Preference Choices

\begin{tabular}{|c|c|c|c|c|c|c|c|c|c|c|}
\hline \multirow[b]{3}{*}{ Item $^{\mathrm{a}}$} & \multicolumn{6}{|c|}{ Frequencies of Left/Right Preferences } & \multicolumn{4}{|c|}{ Distribution Differences } \\
\hline & \multicolumn{2}{|c|}{ Video } & \multicolumn{2}{|c|}{ Surface } & \multicolumn{2}{|c|}{ Wire Frame } & \multicolumn{2}{|c|}{ Surface/Video $^{\mathrm{b}}$} & \multicolumn{2}{|c|}{ Wire Frame/Video ${ }^{b}$} \\
\hline & $\overline{f_{\text {left }}}$ & $\overline{f_{\text {right }}}$ & $\overline{f_{\text {left }}}$ & $\overline{f_{\text {right }}}$ & $f_{\text {left }}$ & $\overline{f_{\text {right }}}$ & $\chi^{2}$ & $p$ & $\chi^{2}$ & $p$ \\
\hline sympathy & 30 & 2 & 24 & 9 & 26 & 7 & 1.56 & .212 & 0.79 & .375 \\
\hline activity & 20 & 12 & 9 & 25 & 16 & 17 & 7.06 & $.008^{* * *}$ & 1.04 & .308 \\
\hline dominance & 9 & 23 & 13 & 20 & 15 & 17 & 1.49 & .222 & 4.00 & $.046^{*}$ \\
\hline attention & 18 & 11 & 22 & 10 & 19 & 12 & 0.23 & .631 & 0.00 & .956 \\
\hline perspective & 27 & 5 & 23 & 10 & 22 & 11 & 0.84 & .359 & 1.23 & .268 \\
\hline identify with & 27 & 3 & 19 & 13 & 23 & 9 & 3.33 & .068 & 1.17 & .280 \\
\hline talk to & 29 & 3 & 28 & 6 & 25 & 8 & 0.26 & .612 & 0.80 & .370 \\
\hline
\end{tabular}

aShort item labels. See dependent measures for detailed description. ${ }^{\mathrm{b}} d f=1 . \quad{ }^{*} p \leq .05 . \quad{ }^{* *} p \leq .01$.

right person did show more body movement activity (percent of time moving) than the person on the left.

\section{DISCUSSION}

A platform for the 3-D computer animation of body movement has been introduced as a new tool for nonverbal communication research. In contrast to previous approaches, which were limited either with respect to the range of simulated behavior or with respect to the realism of the computer animations, the new instrument allows for sophisticated 3-D animations of whole body movement using realistic 3-D polygon models with skeletons and skinlike envelopes. By means of a special converter program, it has been made possible to translate so-called position time-series protocols of videorecorded movement behavior into animation scripts of a professional computer animation platform. The new method was evaluated in an experiment comparing person perception effects of videotaped nonverbal interactions with computer animations of the same behavior to find out whether similar impression effects could be obtained. A significant difference could be found in the focus of visual attention, which shifted in the computer animation modes from the head area to the hand area. This difference can probably be attributed to the lack of facial activity in the computer models. Although the focus of visual attention changed, the results indicate a remarkable correspondence between the impression ratings based on video recordings and those based on computer animations. This correspondence not only provides a strong argument for the capacity of our animations to accurately convey the relevant movement cues but also leads to the assumption that a considerable part of the variance in the impression data is explained by the observed movement behavior. As shown, these results are very robust against variations in the social setting of the experiment (group vs. single subject) and display technique (HMD vs. large-screen LCD projector). In addition, the realism of the computer models did not make any difference with respect to the impression ratings.

Overall, the data suggest that the use of the computer animation paradigm in nonverbal research deserves further attention. Future studies should focus on the inter- personal variance in nonverbal behavior and will have to show that person perception effects induced by video recordings can be reproduced by computer animations, even if the portrayed interaction is less dramatic than in our experiment and the nonverbal behavior is more subtle. Research can now focus on the experimental variation of specific nonverbal cues. The availability of a protocol language that is easily understandable for the human observer and that can also be converted into a motion script for a 3-D animation program is essential for this approach. Thus it is possible to modify particular behavioral aspects interactively by altering the correspondent entry in the movement database and then generate a new animation sequence showing identical behavior except for the nonverbal cue under investigation. With respect to the generation of the database, time-consuming video transcription can be replaced by automatic behavior registration using full-body motion capture equipment. Although the data formats delivered by these devices are compatible with most professional animation programs, it has to be pointed out that these formats are hard to interpret on the phenomenological level. Thus, even in the case of automatic registration, a digital representation of the behavior is needed that can be decoded by the computer and the human observer. Position timeseries protocols such as those used in our studies seem to be an adequate digital representation of movement behavior in both respects since they allow for the interactive editing of behavior protocols as well as the generation of realistic computer animations.

\section{Availability}

More information about the presented program is available by sending a self-addressed envelope with return postage to G.B. Source codes and compiled versions of the program (exe-files) can be obtained by enclosing a recordable $\mathrm{CD}$. Using ICARUS requires the animation tool SoftImage3D and a Pentium III workstation.

\section{REFERENCES}

ARgYle, M. (1972). Non-verbal communication in human social interaction. In R. A. Hinde (Ed.), Nonverbal communication (pp. 243269). London: Methuen.

Bente, G. (1989). Facilities for the graphical computer simulation of head and body movements. Behavior Research Methods, Instruments, \& Computers, 21, 455-462. 
Bente, G., Feist, A., \& Elder, S. (1996). Person perception effects of computer-simulated male and female head movement. Journal of Nonverbal Behavior, 20, 213-228.

Bente, G., Petersen, A., \& Krämer, N. C. (1999). Virtuelle Realität und parasoziale Interaktion: Entwicklung eines Verfahrens zur Untersuchung sozioemotionaler Effekte computersimulierten Verhaltens [Virtual reality and parasocial interaction: A tool for analyzing socioemotional effects of computer-simulated behavior] (Research report). University of Cologne.

BERRY, D. S. (1990). The perceiver as naive scientist or the scientist as naive perceiver? An ecological view of social knowledge acquisition. Contemporary Social Psychology, 14, 145-153.

Berry, D. S., Kean, K. J., Misovich, S. D., \& Baron, R. M. (1991) Quantized displays of human movement: A methodological alternative to the point-light display. Journal of Nonverbal Behavior, 15, 81 97.

Buschmann, J.-U., \& KRÄMER, N. C. (1999). IKARUS Interaktives Kodier-, Analyse, Rendering- und Simulationsmodul. Software Manual [ICARUS Interactive Coding, Analyzing, and Rendering Utility Software. Software Manual] (Research report). University of Cologne.

DAvIS, M. (1972). Understanding body movement: An annotated bibliography. New York: Arno.

Frey, S., Hirsbrunner, H. P., Florin, A., Daw, W., \& Crawford, R. (1983). A unified approach to the investigation of nonverbal and verbal behavior in communication research. In W. Doise \& S. Moscovici (Eds.), Current issues in European social psychology (pp. 143-199). Cambridge: Cambridge University Press.

Frey, S., \& Pool, J. (1976). A new approach to the analysis of visible behavior (Research report). Berne, Switzerland: University of Berne, Department of Psychology.

Grammer, K., Kruck, K. B., \& Magnusson, M. S. (1998). The courtship dance: Patterns of nonverbal synchronization in oppositesex encounters. Journal of Nonverbal Behavior, 22, 2-29.

Harmon, L. D. (1973). The recognition of faces. Scientific American, 229, 71-82.

Hirsbrunner, H. P., Frey, S., \& Crawford, R. (1987). Movement in human interaction: Parameter formation and analysis. In A. W. Sieg- mann \& S. Feldstein (Eds.), Nonverbal behavior and communication (pp. 99-140). Hillsdale, NJ: Erlbaum.

KEMPTER, G. (2000). Trait attribution to reanimated gestural movements. Manuscript submitted for publication.

Krohne, H. W., Egloff, B., Kohlmann, C.-W., \& Tausch, A. (1996). Untersuchungen mit einer deutschen Version der "Positive and Negative Affect Schedule" (PANAS) [Investigations with a German version of the "Positive and Negative Affect Schedule" (PANAS)]. Diagnostica, 42, 139-156.

LeUsCHNer, H. (1999). Vom Videotranskriptzur Computeranimationnonverbalen Verhaltens: Methodendokumentation [From video-transcript protocol to computer animation of nonverbal behavior: Method documentation] (Research report). University of Cologne.

Lewis, R. J., Derlega, V. J., Shankar, A., Cochard, E., \& Finkel, L. (1997). Nonverbal correlates of confederates' touch: Confounds in touch research. Journal of Social Behavior \& Personality, 12, 821 830

Mehrabian, A. (1969). Significance of posture and position in the communication of attitude and status relationships. Psychological Bulletin, 71, 359-372.

Mehrabian, A. (1970). A semantic space for nonverbal behavior. Journal of Consulting \& Clinical Psychology, 35, 248-257.

Morrone, M. C., Burr, D. C., \& Ross, J. (1983). Added noise restores recognizability of coarse quantized images. Nature, 305, 226-228.

Schouwstra, S. J., \& Hoogstraten, J. (1995). Head position and spinal position as determinants of perceived emotional state. Perceptual \& Motor Skills, 81, 673-674.

Trautner, H. M. (1991). Children's and adults' awareness of sexstereotyped postures. Paper presented at the Eleventh Biennial Meeting of the ISSBD, Minneapolis.

Watson, D., Clark, L. A., \& Tellegen, A. (1988). Development and validation of brief measures of positive and negative affect: The PANAS scales. Journal of Personality \& Social Psychology, 54, 1063-1070.

(Manuscript received October 5, 2000; accepted for publication May 16, 2001.) 